\title{
Score Level Fusion for Fingerprint, Iris and Face Biometrics
}

\author{
Ashraf Aboshosha \\ NCRRT, Atomic Energy \\ Authority \\ Nasr City, Cairo, Egypt
}

\author{
Kamal A. El Dahshan \\ Faculty of Science, Al-Azhar \\ University \\ Nasr City, Cairo, Egypt \\ Ebeid A. Ebeid \\ Faculty of Science, Al-Azhar University \\ Nasr City, Cairo, Egypt
}

\author{
Eman A. Karam \\ Girl's College of Science, Al- \\ Azhar University \\ Nasr City, Cairo, Egypt
}

\begin{abstract}
Single biometric systems suffer from many challenges such as noisy data, non-universality and spoof attacks. Multimodal biometric systems can solve these limitations effectively by using two or more individual modalities. In this paper fusion of fingerprint, iris and face traits are used at score level in order to improve the accuracy of the system. Scores which obtained from the classifiers are normalized first using min$\max$ normalization. Then sum, product and weighted sum rules are used to get fusion. Experimental results show that multimodal biometric systems outperform unimodal biometric systems and weighted sum rule gives the best results comparing with sum or product method.
\end{abstract}

\section{Keywords}

Fusion, multimodal, fingerprint recognition, iris recognition, face recognition.

\section{INTRODUCTION}

Biometric means identity verification of persons according to their physical or behavioral characteristics. Many physical body parts and personal features have been used for biometric systems: fingers, hands, irises, faces, ears, voices, gaits, odors, feet, signatures, and DNA. Person verification based on biometric features has attracted more attention in designing systems [1]. Most of biometric systems are far from satisfactory in terms of user confidence and user friendliness and have a false rejection rate (FRR). There is a need to develop novel algorithms for human recognition. Multimodal biometric systems use multiple modalities to overcome the limitations that arise when use single biometric trait such as: noise, non universality, lack of individuals and spoof attacks. Multimodal biometric systems perform better than unimodal biometric systems [2]. In our work three public and robust biometrics are used"fingerprint, iris and face". In first stage image preprocessing is performed on fingerprint, iris and face images using different techniques for each biometric. In the second stage three feature extraction techniques are applied: Minutia based algorithm are used for fingerprint which extracts two types of points, ridge ending and ridge bifurcation [11]. Modified Daugman's algorithm is used for iris recognition where enhanced iris image is segmented first to localize circular iris and pupil region, The extracted iris region was then normalized into a rectangular block with constant dimensions to account for imaging inconsistencies. Finally, the phase data from 1D Log-Gabor filters were extracted and quantized to four levels to encode the unique pattern of the iris into a bit-wise biometric template using Daugman's rubber sheet model [12],[13]. Local binary pattern LBP is performed for face images where face image is divided into cells then for each cell an 8-digit binary number is computed which converted to decimal form then histogram is computed over cells, finally all histograms are concatenated to give feature vector [14]. In the third stage: matching scores from each matcher are arrived, then these scores are normalized by min-max rule to convert scores between 0 and 1 [4]. In the fourth stage: fusion at score level is performed where normalized scores are combined using sum and product rules. The objective of this research is as follow: First designing and implementing monomodal systems for the biometric recognition of fingerprint, iris and face; second designing and implementing a multimodal biometric system of the combined biometrics by fusing the scores that arise from each matcher. Three biometrics are used here to give high degree of discrimination when we have large number of users or population. Third: carrying out intensive tests on the fingerprint, iris and face databases using the proposed schemes to conclude the best of them. The paper is organized as follows: in section 2 related works are presented; in section 3 previous works in fingerprint, iris and face recognition systems are given; in section 4 state of the art of multimodal biometric; in section 5 the research methodology are presented; the experimental results and analysis are reported in section 6; conclusion is given in the last section.

\section{RELATED WORK}

Different literatures can be founded which present variety of approaches for unimodal and multimodal biometric systems. Multimodal biometrics has been proposed by Ross and Jain in 2003 [3]. regarding fingerprint, iris and face biometrics fusion any pair of them " fingerprint and iris ", " iris and face" or "fingerprint and face" has attracted a lot of attention and different researches have proposed many of approaches. Maryam et al. [4] in 2012 proposed fusion of face and iris to obtain a robust recognition system .in That study the proposed method use Local Binary pattern local feature extractor and subspace linear discriminant analysis global feature extractor on face and iris respectively. Face and iris scores are normalized using tanh normalization, then Weighted sum rule is applied for the fusion. Experimental results which performed on ORL, FERET, CASIA and UBIRIS databases show that multimodal biometric system outperform single biometric system. Yang and Fan [5] in 2007 used fingerprint, palmprint and hand geometry to implement personal identity verification. these three biometric traits can be taken from the same image. they perform matching score fusion at different levels to establish person, performing a first fusion of the fingerprint and palm print features, and then a matching score fusion between the multimodal system and hand geometry system .the system was tested on a database containing the features self-constructed by 98 subject. Mohamed et al. [6] in 
2013 multimodal biometric system fusion using fingerprint and iris are proposed, decision level is used for fusion and each biometric result is weighted for participate in final decision .fuzzy logic is used for the effect of each biometric result combination. The proposed method has achieved high accuracy comparing with unimodal systems. Byungium and Yillbyung [7] in 2005 were presented biometric authentication system based on iris and face, they applied 2-D discrete wavelet transform to extract the feature sets from iris and face. and then Linear Discriminant Analysis is applied to obtain reduced joint feature vector from these feature sets. databses which used to show experimental results are ORL for face images, and for iris database the images are acquired through CCD camera with LED lamp around lens under indoor light. Ajita and Massimo [9] in 2009 addressed the feature level fusion of multi-modal and multi-unit sources of information by proposing approach computes the SIFT features from both biometric sources.for each biometric trait feature selection on the extracted SIFT features was performed by spatial sampling then the features are concatenated to form a single vector using serial fusion. L.Latha and S.Thangasamy [10] in 2010 they have used left and right irises and retinal features, and after matching process the scores are combined using weighted sum rule. To validate their approach, experiments were conducted on the iris and retina images obtained from CASIA and VARIA database respectively.

\section{FINGERPRINT RECOGNITION}

A fingerprint is the feature pattern of one finger. It is believed with strong evidences that each fingerprint is unique. Each person has his own fingerprints with the permanent uniqueness. So fingerprints have being used for identification and forensic investigation for a long time [15]. Fingerprint is composed of ridges and furrows which are parallel and have the same width. In fingerprint recognition, fingerprint is distinguished by minutiae, which are points on the ridges. There are many types of minutia, but the two basic types are: termination which represent the ending of the ridge and the other is called bifurcation which is the point of the ridge from which two branches derive [16], [6] as in figure (1).
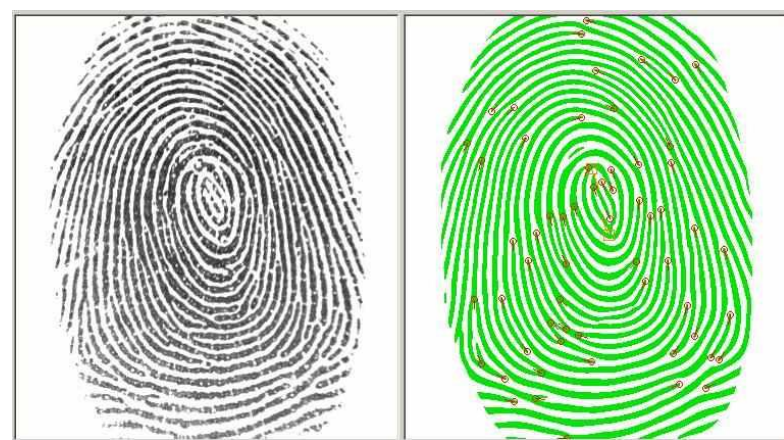

Fig 1: Fingerprint image and minutia points

Fingerprint identification system has three parts as follow:

\subsection{Fingerprint Acquisition}

Image capture devices include different categories of fingerprint capture devices, such as: optical, solid-state and ultrasonic [11]. Optical fingerprint capture devices have the longest use history of these categories.

\subsection{Feature Extraction}

In this work minutia based algorithm are used, in this method, the resulting feature vector is containing for each minutia point the following parameters: 1) x-coordinate, 2) y- coordinate, and 3) orientation. Feature extraction part can be summarized as follow:

\subsubsection{Preprocessing}

Histogram equalisation and Fast Fourier transform (FFT) are used for image enhancement [17]. Histogram equalisation is employed to expand the pixel value distribution of an image so as to increase the perceptional information. FFT is used to connect false broken points of ridges and increase the contrast between ridges and furrows. Binarization is then performed using locally adaptive thresholding to transform the 8-bit grayscale fingerprint image into a binary image where 0 s indicate ridges and $1 \mathrm{~s}$ furrows. Image segmentation is achieved through a three step approach: (1) block direction estimation, (2) segmentation by direction intensity and (3) morphological open and close operations to extract regions of interest (ROI)

\subsubsection{Minutia Extraction}

Befor minutia extraction, Ridge thinning is performed first to remove the redundant pixels of ridges till the ridges wide is just one pixel. Now fingerprint image is ready to extract minutia. The simple algorithm for minutia extraction is: if a pixel with 1 value has one neighbor with 1 value in its 8 neighbors, it is terminate and if it has three neighbors with 1 value it is bifurcation [6], [18].

\begin{tabular}{|r|r|r|}
\hline 0 & 1 & 0 \\
\hline 0 & 1 & 0 \\
\hline 1 & 0 & 1 \\
\hline
\end{tabular}

\begin{tabular}{|r|r|r|}
\hline 0 & 0 & 0 \\
\hline 0 & 1 & 0 \\
\hline 0 & 0 & 1 \\
\hline
\end{tabular}

\section{Fig 2: The left form is bifurcation and the right is} termination

\subsubsection{Post-processing}

This phase is to remove false minutia to reduce the complexity of computation and enhance the accuracy of the system. The false minutia are defined as seven types, most of them can be removed by proposing a threshold $\mathrm{D}$, if the distance between minutiae less than $\mathrm{D}$, these minutiae will be removed [18].

\subsection{Fingerprint Matching}

An alignment-based match algorithm includes two consecutive phases: first is alignment phase and the second is match phase. In alignment phase each set of minutia is transformed to a new coordination system with the referenced points to coincident with the direction of the referenced points. For each fingerprint, translate and rotate all other minutia with respect to the references minutia according to the following formula:

$$
\left(\begin{array}{l}
x_{i}-\text { new } \\
y_{i}-n e w \\
\theta_{i}-n e w
\end{array}\right)=T M *\left[\begin{array}{c}
x_{i}-x \\
y_{i}-y \\
\theta_{i}-\theta
\end{array}\right]
$$

Where $\left(\mathrm{x}_{i}, \mathrm{y}_{i}, \theta_{i}\right)$ is the parameters of the reference minutia and

$$
T M=\left(\begin{array}{ccc}
\cos \theta & -\sin \theta & 0 \\
\sin \theta & \cos \theta & 0 \\
1 & 1 & 0
\end{array}\right)
$$


the last phase is the matching process, where we use elastic match algorithm to count the matched minutia pairs of two fingerprint images. In this method bounding box around each template minutia is assumed. If the minutia to be matched is within the rectangle box and the direction discrepancy between them is very small, then the two minutias are regarded as a matched minutia pair. Each minutia in the template image either has no matched minutia or has only one corresponding minutia [18].

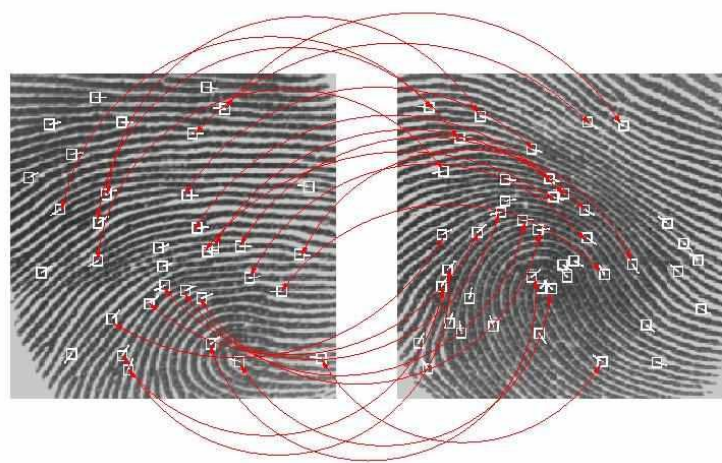

Fig 3: Matching process between minutiae pairs

\section{IRIS RECOGNITION}

The iris system composes of a number of subsystems, which correspond to each stage of iris recognition. The stages include segmentation for locating the iris region in an eye image, normalization for creating a dimensionally consistent representation of the iris region, enhancement by histogram equalization of normalized iris region, feature encoding for creating an iris code containing only the most discriminating features of the iris and finally matching by hamming distance to make a decision of acceptance or rejection. See figure (4)

\subsection{Iris Segmentation}

Segmentation is the first stage in iris preprocessing to isolate the required iris region from the whole eye image by separating the part of an image between the inner boundary and outer boundary. Canny method used to detect edges by searching about local maxima of the gradient of iris image. The gradient is computed using the derivative of a Gaussian filter. It determines two values as thresholds to reveal strong and weak edges. This method is more robust to noise and more likely to detect true weak edges. The output of the canny edge detector is the edge strength image and the orientation image. The image intensity value has to be increased by adjusting the gamma correction factor. With the orientation image and the adjusted gamma image as the input, the local maxima are suppressed. Then Circular Hough Transform is used to detect the iris and pupil boundaries and reveal both radius and center coordinates [10].

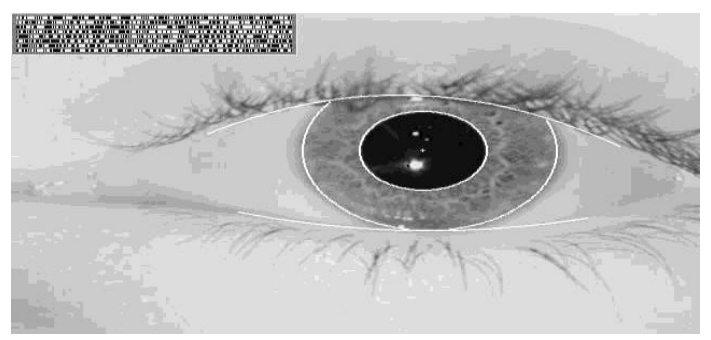

Fig 5: Iris segmentation

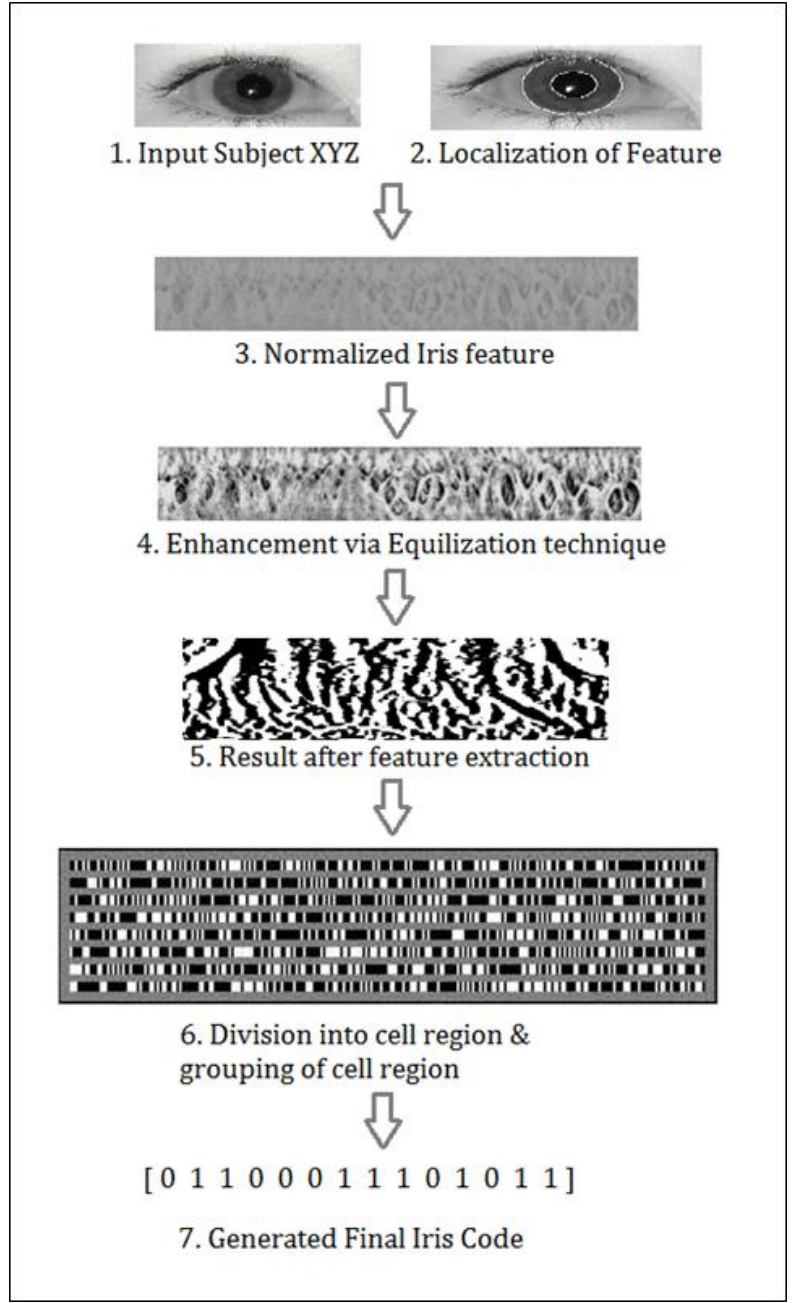

\section{Fig 4: The different phases for iris recognition}

\subsection{Iris Normalization}

Normalization is a process of transforming the segmented iris region into fixed dimension. However, dimensional inconsistencies arise between eye images due to the stretching of iris, caused by pupil dilation from varying levels of illumination. Such elastic distortion in iris texture will affect the result of iris matching. Therefore, normalization is done to recover the iris deformation caused by illumination variations. The normalization process uses Daugman's rubber sheet model and this method remaps the annular iris image $\mathrm{I}(\mathrm{x}, \mathrm{y})$ from original Cartesian coordinates $(\mathrm{x}, \mathrm{y})$ to a dimensionless pseudo polar coordinate system I $(r, \Theta)$. Rubber sheet model takes into account the pupil dilation and size inconsistencies in order to produce a normalized representation with constant dimension. Normalization produces a 2D array with horizontal dimensions of angular resolution and vertical dimensions of radial resolution. A template of dimension $20 \mathrm{x}$ 240 is produced, where 20 are the radial resolution and 240 is the angular resolution [19], [20].

\subsection{Iris Enhancement}

Applying histogram equalization (HE) improves the contrast of the image by enhancing the normalized pattern. Equalization implies mapping one distribution (the given histogram) to another distribution. HE enhances the global contrast of image, when the pixel values of the image are represented by Convergent contrast values. In this process, the intensity values can be better distributed on the histogram by redistributing the most frequent intensity values. This action 
pushes the areas of lower local contrast to gain a higher contrast without affecting the global contrast.

\subsection{Feature Encoding}

Feature encoding extracts the underlying information from the iris pattern and generates the binary iris template that is used in matching. Convolving the normalized iris pattern with 1D Log-Gabor filter generates the iris feature set. The filter is Gaussian on a logarithmic scale and used to produce zero DC components for any bandwidth. It is given by:

$$
G(f)=\exp \left(\frac{-\log \left(\frac{f}{f_{o}}\right)^{2}}{2 \log \left(\frac{\sigma}{f_{o}}\right)}\right)
$$

Where $\left(f_{o}\right)$ represents the centre frequency, and gives the bandwidth of the filter. By applying 1D Log-Gabor filter, the 2D normalized pattern is divided into a number of $1 \mathrm{D}$ signals and these are convolved with 1D Gabor wavelets. The rows of the 2D normalized pattern are taken as the 1D signal; each row corresponds to a circular ring on the iris region. The angular direction is taken, which corresponds to columns of the normalized pattern, since maximum independence occurs in the angular direction. The filter is constructed by calculating the radial filter component such as center frequency of filter and normalized radius from the center of frequency plan. The resultant complex features are phase quantized and encoded into binary iris templates [21], [24].

\subsection{Iris Matching}

Matching is a process to determine whether two iris templates are from the same individual or not. Hamming distance is applied for bit-wise comparisons of images. Noise in the iris image is masked and only significant bits generated from the true iris region are used in the Hamming distance calculation between two iris templates [22], [23].

$$
H D=\frac{\|(\operatorname{code} \mathrm{A} \otimes \operatorname{code} \mathrm{B}) \mathrm{I}(\operatorname{mask} \mathrm{A} \text { I mask } \mathrm{B}) \|}{\|\operatorname{mask} \mathrm{A} \mathrm{I} \operatorname{mas} k \mathrm{~B}\|}
$$

where $H D$ is the Hamming distance, $\mathrm{A}$ and $\mathrm{B}$ are two normalized iris images, code $\mathrm{A}$ and code $\mathrm{B}$ are the bit-codes of $\mathrm{A}$ and $\mathrm{B}$, mask $\mathrm{A}$ and mask $\mathrm{B}$ are respectively the masks of noise of A and B which produced by eyelashes or eyelids. The hamming distance between the templates, which have deployed the best bits is reduced comparing with the use of full iris code. If two irises are identical then $H D$ will give 0 results.

\section{FACE RECOGNITION}

Two decades ago face recognition has became an important topic in computer vision. This is due it has potential application values [25]. A lot of approaches have been presented to solve face recognition problems. Principal Component Analysis (PCA) [26], and Linear Discriminant Analysis (LDA) [27] based methods, has significantly face recognition methods. In PCA, a face subspace is formed to represent optimally only the face; by using LDA, a discriminant subspace is formed to discriminate faces of different subjects. Gabor wavelet based and Local features analysis are other approaches which build a local appearancebased feature space, these approaches are more robust against various changes by using appropriate image filters[28]. Local Binary Patterns (LBP) is presented as a Strong local descriptor for microstructures of images [29]. In this work local binary pattern are used for face recognition.

\subsection{Face recognition with LBP}

The original LBP operator was introduced by Ojala et al. [30]. It is a powerful means of texture description. The face area is first divided into small regions from which Local Binary Pattern (LBP) histograms are extracted and concatenated into a single vector see fig (6)

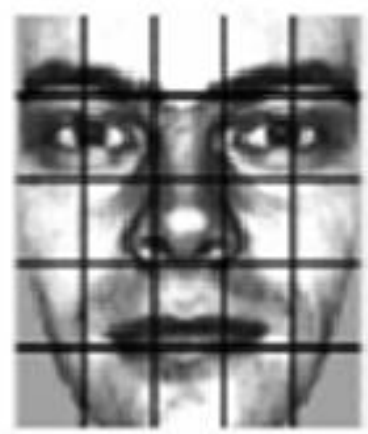

Fig 6: Facial image divided into $5 \times 5$ regions

In each region the operator labels the pixels of an image by threshold the $3 \times 3$-neighbourhood of each pixel with the center value and considering the result as a binary number or a decimal number.

$$
\begin{aligned}
& \boldsymbol{L B P}=\sum_{p=0}^{p-1} s\left(f(x, y)-f\left(x_{p}, y_{p}\right)\right) 2^{p} \\
& \boldsymbol{S}(\boldsymbol{Z})= \begin{cases}1 & \text { if } \quad z \geq 0 \\
0 & \text { if } \quad z<0\end{cases}
\end{aligned}
$$

Then the histogram of the labels can be used as a texture descriptor. Figure (7) illustrate the original LBP operator. Later the operator was extended to use neighborhoods of different sizes .Using circular neighborhoods and bilinearly interpolating the pixel values allow any radius and number of pixels in the neighborhood. For neighborhoods the notation $(\mathrm{P}, \mathrm{R})$ are used which means $\mathrm{P}$ sampling points on a circle of radius of R. See Figure (8) as an example of the circular $(8,2)$ neighborhood.

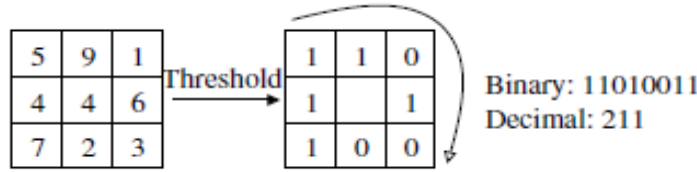

Fig 7: Basic LBP operator

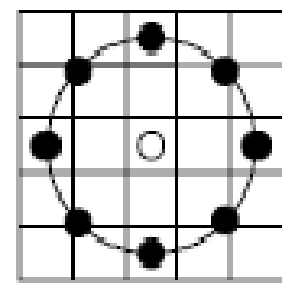

Fig 8: Circular $(8,2)$ neighborhood 
Another modification to the original operator uses so called uniform patterns [30], [31]. A Local Binary Pattern is called uniform if it contains at most two bitwise transitions from 0 to 1 or vice versa when the binary chain is considered circular. For example, 11100000, 00011110 and 11000001 are uniform patterns. Ojala et al. noticed that in their experimental results with texture images, uniform patterns account for a bit less than $90 \%$ of all patterns when using the $(8,1)$ neighborhood and for around $70 \%$ in the $(16,2)$ neighborhood. Shengcai Liao et al. proposed an improved method over the basic LBP in which multi-scale block LBP are used. Multiscale LBP is an extension to the basic LBP, with respect to neighborhoods of different sizes. In MB-LBP, the comparison operator between individual pixels in LBP is simply replaced with comparison between average gray-values of sub-regions. Each sub-region is a square block containing neighboring pixels (or just one pixel particularly). The whole filter is composed of 9 blocks. We take the size s of the filter as a parameter, and $\mathrm{s} \mathrm{x}$ $\mathrm{s}$ denoting the scale of the MB-LBP operator (particularly, $3 \times 3 \mathrm{MB}-\mathrm{LBP}$ is in fact the original LBP). Note that the scalar values of averages over blocks can be computed very efficiently from the summed-area table or integral image [25]. For this reason, MB-LBP feature extraction can be very fast, and it only incurs a little more cost than the original $3 \times 3$ LBP operator [14]. Other different version of LBP which outperform the original LBP are proposed by researches like completed LBP (CLBP), dominant LBP (DLBP) and LBP Histogram Fourier (LBP-HF). For matching two facial images there are several possible dissimilarity measures have been proposed for histograms [32].Histogram intersection:

$$
D(S, M)=\sum_{i} \min \left(S_{i}, M_{i}\right)
$$

Log-likelihood statistic:

$$
L(S, M)=-\sum_{i} S_{i} \log M_{i}
$$

Chi square statistic:

$$
\chi^{2}(S, M)=\sum_{i} \frac{\left(S_{i}-M_{i}\right)^{2}}{S_{i}+M_{i}}
$$

Where $\mathrm{S}$ and $\mathrm{M}$ represent the matched face images, in our work chi square static measure have been used.

\section{MULTIMODAL BIOMETRICS SYSTEMS}

Multibiometric systems have five different methods to address problems associated with single biometric systems [6]. Figure (9) show these types

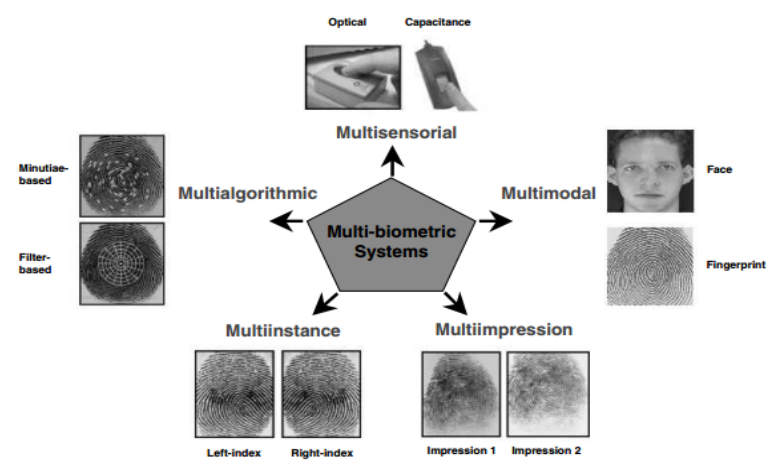

\subsection{Multi Sensor}

Two or more sensors are used to obtain data from one biometric trait such as fingerprint image with optical and ultrasound sensors and facial image by visible light camera or infrared camera.

\subsection{Multi Representation}

Several sensors capturing several similar body parts (multi fingerprint image from multi finger but from one person).

\subsection{Multi Instance}

The same sensor captures several instances of the same body part. For example, system capturing images from multiple fingers are considered to be multi-instance.

\subsection{Multi Algorithm}

Two or more of different algorithms are used for the same trait. Maximum benefit would be derived from algorithms that are based on different and independent principles.

\subsection{Multi Modal}

Using two or more of different biometric traits which captured from different sensors and employ them in the variety fusion strategies.

\section{FUSION STRATEGIES}

Feature level: The data obtained from sensor is used to extract the feature vector from one biometric trait which are independent from those extracted from the other, these feature vectors are concatenated to produce a single new vector. This process is difficult when feature vectors are heterogeneous.

Matching score level: Each system provides a matching score indicating the nearness of the feature vector with the template vector. These scores can be combined to assert the veracity of the claimed identity [33]. While the information contained in matching scores is not as rich as in images or features, it is much richer than ranks and decisions. Further, it is easier to study and implement than image-level and feature-level fusion. It can also be used in all types of biometric fusion scenarios. Decision level: Each individual biometric process gives its own binary result. The fusion process fuses them together to outputs single binary decision accepts or reject.

\section{NORMALIZATION}

Normalization score process aims to bring score values in the interval $[0,1]$ in order to execute the score fusion, many of normalization methods are presented. Min-max method is one of them, it is given by the following form respect to fingerprint:

$$
N_{\text {finger }}=\frac{M S_{\text {finger }}-\text { min }_{\text {finger }}}{\text { max }_{\text {finger }}-\text { min }_{\text {finger }}}
$$

Iris and face normalization are the same where MS is the matching score obtained from the matcher, min and max are the minimum and maximum score values respectively. Other normalization rules have been given in verity researches like Z-score and Tanh method. Both min-max and Z-score are simple but they are sensitive to presence of outliers in score matrix, Tanh method is more efficiency but involves a lot of parameters.

Fig 9: Multimodal types 


\section{RESEARCH MEYHODOLOGY}

The different stages of our multimodal biometric system are being shown in figure (10), these stages are executed as follow:

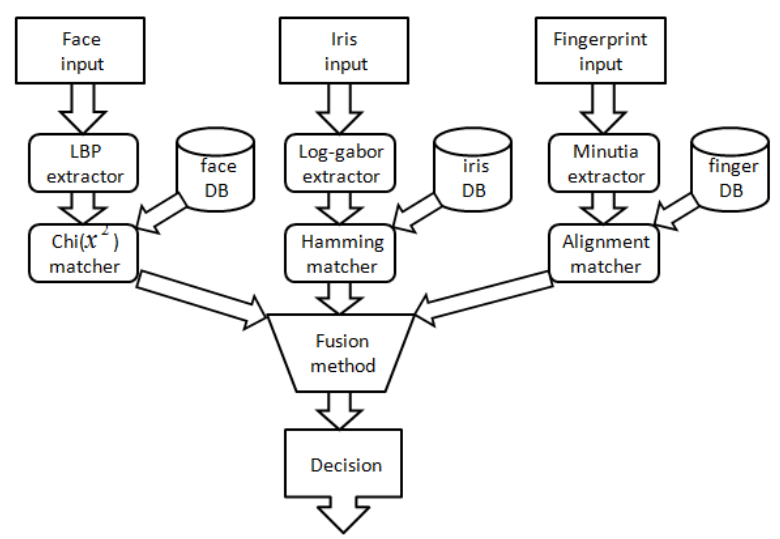

Fig 10: Proposed multimodal stages

Acquisition Images: In this stage fingerprint, iris and face image are captured by appropriate sensor for each trait then the three images are saved to be the input in next step.

Feature Extraction: This is the second stage where three feature extraction algorithms are presented to extract and form the feature template. Minutia-based algorithm are applied to extract feature from finger image, Daugman algorithm with 1D log-Gabor filter for iris template extraction and Local Binary Pattern (LBP) to extract feature from face image.

Matching scores: Each extracted template is matched with the corresponding templates in the database. An alignment-based match algorithm is used as fingerprint matcher who determines the similarity between fingerprint templates, Hamming distance HD is applied for iris matching stage to give the dissimilarity between iris images and Chi square for face matching process which introduces the dissimilarity between face images.

Normalization: Where the matching scores which result from different classifiers have a different domain, so it must be normalized first before fusion phase, min-max normalization method are used here to give a unified domain for all score values.

Fusion: In this stage normalized matching scores are fused to output one from three scores. Sum, product and average rules are applied in our work as follow:

Sum rule:

$$
f_{i}=\sum_{m=1}^{M} S_{i}^{m}, \forall i
$$

Product rule:

$$
f_{i}=\prod_{m=1}^{M} S_{m}^{i}, \forall i
$$

Where $m=1,2,3 \ldots, \mathrm{M}, \mathrm{M}$ is the number of matchers applied to individual $i$ where $i=1,2 \ldots$. I. I is number of individuals.

Weighted sum rule:

$$
f_{i}=\sum_{m=1}^{M} W_{m} * S_{i}^{m}, \forall i
$$

Where $\mathrm{W}_{m}$ is the weights associated with each individual score.

Decision: Finally, Boolean function can be used to result binary decision in order to identify the person if he is genuine or impostor.

\section{EXPEREMENTAL RESULTS}

Three sets of databases are used to evaluate the performance of unimodal and multimodal systems. The first is FVC2004 DB3_A database for fingerprint recognition which contains grey scale images and TIF files, it contains 100 different subject each of them has 8 samples, three samples are selected for training and the rest samples are used for testing. CASIA database are prepared for iris recognition which is gray scale images and JPEG file, three samples are used for training and the remaining five images served as testing images. The recognition was done on 100 subject picked randomly from database. The third database is the face database which collected from face94 (university of Essex, UK). Every person has 20 samples and some individuals are wearing glasses and beards with different facial expressions. It contains images of JPEG files and colored. We have chosen 100 different persons with 8 samples; the first 3 samples are used for training and 5 for testing. All these databases are independent from each other because there is no common database contains three biometrics" fingerprint, iris and face" for the same person.

\subsection{Experimental on Unimodal System}

In this work three unimodal biometric systems are given" fingerprint, iris and face", to determine the accuracy of the systems. Two measures are selected: false accept rate (FAR) and false reject rate (FRR) which they are computed on the given databases. Where false accept rate (FAR) is the probability that the system incorrectly matches the input pattern to a non-matching template in the database. It measures the percent of invalid inputs which are incorrectly accepted. False reject rate (FRR) is the probability that the system fails to detect a match between the input pattern and a matching template in the database. It measures the percent of valid inputs which are incorrectly rejected [34]. The aim is always to reduce both FAR and FRR to get better accuracy. In table 1: FVC2004 DB3_A database is collected to evaluate fingerprint recognition systems by using minutia-based algorithm, the best accuracy given by the system equal 82.5 $\%$. Table 2 gives experimental results on CASIA database to evaluate iris recognition system which use log-Gabor filter algorithm; the best accuracy achieved by the system is 93.15\%. Table 3 presents experimental results on face94 (university of Essex, UK) database to evaluate face recognition system which use local binary pattern algorithm, the system gives accuracy equal to $97.58 \%$.

Table 1. Some of threshold values and the corresponding FAR and FRR indexes in fingerprint recognition system.

\begin{tabular}{|c|c|c|}
\hline Threshold & FAR \% & FRR \% \\
\hline 31 & 25.00 & 14.21 \\
\hline 32 & 19.51 & 16.63 \\
\hline 33 & 17.80 & 17.52 \\
\hline
\end{tabular}




\begin{tabular}{|l|c|c|}
\hline 34 & 15.73 & 20.00 \\
\hline $\mathbf{3 5}$ & $\mathbf{1 3 . 2 0}$ & $\mathbf{2 2 . 0 0}$ \\
\hline 36 & 12.41 & 24.12 \\
\hline 37 & 11.60 & 26.25 \\
\hline 38 & 8.72 & 29.10 \\
\hline 39 & 7.30 & 32.21 \\
\hline 40 & 6.31 & 36.00 \\
\hline
\end{tabular}

Table 2. Some of threshold values and the corresponding FAR and FRR values in iris recognition system.

\begin{tabular}{|c|c|c|}
\hline Threshold & FAR \% & FRR \% \\
\hline 0.73 & 0.00 & 27.67 \\
\hline 0.38 & 0.13 & 23.83 \\
\hline 0.39 & 0.13 & 20.00 \\
\hline 0.40 & 0.25 & 16.96 \\
\hline 0.41 & 0.25 & 13.66 \\
\hline $\mathbf{0 . 4 2}$ & $\mathbf{1 . 5 3}$ & $\mathbf{1 2 . 1 4}$ \\
\hline 0.43 & 4.48 & 9.90 \\
\hline 0.44 & 7.80 & 7.41 \\
\hline 0.45 & 16.90 & 5.62 \\
\hline 0.46 & 33.07 & 4.55 \\
\hline
\end{tabular}

Table 3. Some of threshold values and the corresponding FAR and FRR values in face recognition system.

\begin{tabular}{|c|c|c|}
\hline Threshold & FAR \% & FRR \% \\
\hline 0.6 & 0.00 & 20.00 \\
\hline 0.7 & 0.00 & 16.50 \\
\hline 0.8 & 0.00 & 9.60 \\
\hline 0.9 & 0.25 & 6.50 \\
\hline 1.0 & 0.38 & 5.07 \\
\hline $\mathbf{1 . 1}$ & $\mathbf{0 . 8 9}$ & $\mathbf{3 . 9 2}$ \\
\hline 1.2 & 2.30 & 3.39 \\
\hline 1.3 & 4.60 & 2.76 \\
\hline 1.4 & 7.94 & 2.32 \\
\hline 1.5 & 15.1 & 1.60 \\
\hline
\end{tabular}

\subsection{Experiments on Multimodal System}

Normalized scores which were obtained from fingerprint, iris and face matchers would be combined to give single score for each person. Sum, weighted sum and product rules would be used for fusion process. Tables 4,5 and 6 introduce the experimental results for the proposed platform. From these results we find that sum rule give an accuracy equal to $98.81 \%$, product rule presents accuracy $99.31 \%$, and weighted sum rule give the best accuracy which equal to $99.7 \%$. This means weighted sum rule outperforms sum and product rules. the weights associated with the biometrics are determined experimentally, where the face weight is nearly $0.45 \%$, the iris weight is nearly $0.35 \%$ and the fingerprint weight is $20 \%$.
Table 4. Some of threshold values and the corresponding FAR and FRR values in multimodal biometric system by using sum rule.

\begin{tabular}{|c|c|c|}
\hline Threshold & FAR \% & FRR \% \\
\hline 1.4 & 0.00 & 32.00 \\
\hline 1.5 & 0.00 & 18.00 \\
\hline 1.6 & 0.00 & 8.48 \\
\hline 1.7 & 0.00 & 4.37 \\
\hline $\mathbf{1 . 8}$ & $\mathbf{0 . 5 1}$ & $\mathbf{1 . 8 7}$ \\
\hline 1.9 & 2.30 & 0.98 \\
\hline 2.0 & 7.00 & 0.70 \\
\hline 2.1 & 20.51 & 0.35 \\
\hline 2.2 & 44.71 & 0.17 \\
\hline 2.3 & 71.53 & 0.00 \\
\hline
\end{tabular}

Table 5. Some of threshold values and the corresponding FAR and FRR values in multimodal biometric system by using product rule.

\begin{tabular}{|c|c|c|}
\hline Threshold & FAR \% & FRR \% \\
\hline 0.10 & 0.00 & 6.78 \\
\hline 0.12 & 0.00 & 3.82 \\
\hline 0.14 & 0.00 & 2.50 \\
\hline $\mathbf{0 . 1 6}$ & $\mathbf{0 . 1 2}$ & $\mathbf{1 . 2 5}$ \\
\hline 0.18 & 0.51 & 0.89 \\
\hline 0.20 & 1.92 & 0.80 \\
\hline 0.22 & 3.60 & 0.71 \\
\hline 0.24 & 7.17 & 0.62 \\
\hline 0.26 & 11.92 & 0.62 \\
\hline 0.28 & 15.1 & 0.61 \\
\hline
\end{tabular}

Table 6. Some of threshold values and the corresponding FAR and FRR values in multimodal biometric system by using weighted sum rule.

\begin{tabular}{|c|c|c|}
\hline Threshold & FAR \% & FRR \% \\
\hline 0.53 & 0.00 & 2.41 \\
\hline 0.54 & 0.00 & 1.69 \\
\hline 0.55 & 0.00 & 1.25 \\
\hline 0.56 & 0.00 & 1.10 \\
\hline $\mathbf{0 . 5 7}$ & $\mathbf{0 . 0 0}$ & $\mathbf{0 . 6 1}$ \\
\hline 0.58 & 0.51 & 0.55 \\
\hline 0.59 & 1.28 & 0.55 \\
\hline 0.60 & 2.43 & 0.51 \\
\hline 0.61 & 4.35 & 0.51 \\
\hline 0.62 & 6.79 & 0.50 \\
\hline
\end{tabular}

Figure 11 show the Receiver Operating Characteristic (ROC) curves for the proposed system. ROC curves are obtained by plotting the FAR probability versus the FRR probability with different values of the decision threshold. Equal Error Rate (EER) is the position on the ROC curve where FAR and FRR 
are equal. The ROC curve reveals that the weighted sum rule which have $0.54 \%$ EER is more reliable than product rule with $(0.83 \%$ EER $)$ and sum rule with $(1.20 \%$ EER $)$.

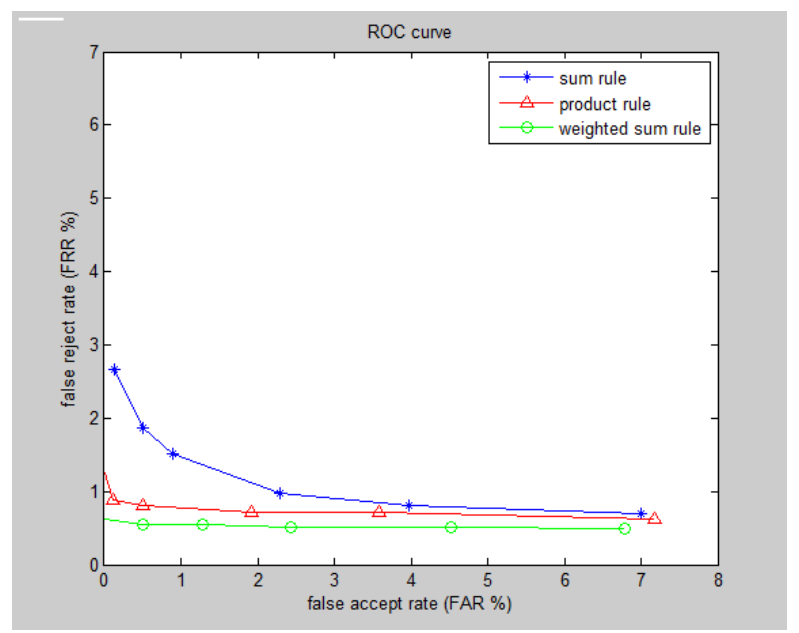

Fig 11: ROC curves of proposed fusion methods on FVC2004 DB3_A, CASIA and faces94 databases

\section{COMPLEXITY OF THE FUSION}

The accuracy of fusion process is computed here in two ways: consumed time and number of required operations

\begin{tabular}{|l|c|c|}
\hline Fusion method & Consumed time & \# Operation \\
\hline sum & 0.040 & 3 \\
\hline product & 0.037 & 3 \\
\hline Weighted sum & 0.055 & 5 \\
\hline
\end{tabular}

The previous table presents the consumed time for one fusion process by microsecond and the number of operations which required in each fusion process per one subject. The weighted sum rule is the most consuming time than sum and product rules. It is easy to discover that the order of system complexity is $O(n)$

\section{CONCLUSION}

Fusion of fingerprint, iris and face systems at score level was proposed. This platform is necessary if we have treat huge databases that contain hundreds millions of users. The scores was normalized first using min-max method then fusion done by using sum, product and weighted sum rules. Each previous method outperforms unimodal systems and increases the total accuracy comparing with these systems. Weighted sum rule outperformed both sum and product rules and presents significantly better results.

In the future we may use other methods to recognize fingerprint, iris and face. These methods will take less computational time and give more accuracy.

\section{REFERENCES}

[1] R Raghavendra, Rao Ashok, and G Hemantha Kumar. 2010. Multimodal biometric score fusion using gaussian mixture model and monte carlo method. Journal of Computer Science and Technology, 25(4):771-782.

[2] Houda Benaliouche and Mohamed Touahria. 2014 Comparative study of multimodal biometric recognition by fusion of iris and fingerprint. The Scientific World Journal, 2014.
[3] A run Ross and Anil Jain. 2004 Multimodal biometrics: An overview. na.

[4] Maryam Eskandari and O“ nsen Toygar. 2012 Fusion of face and iris biometrics using local and global feature extraction methods. Signal, Image and Video Processing, pages $1-12$.

[5] Fan Yang and Baofeng Ma. 2007. A new mixed-mode biometrics information fusion based-on fingerprint, hand-geometry and palm-print. In Image and Graphics, 2007. ICIG 2007. Fourth International Conference on, pages 689-693. IEEE.

[6] Mohamad Abdolahi, Majid Mohamadi, and Mehdi Jafari. 2013. Multimodal biometric system fusion using fingerprint and iris with fuzzy logic. International Journal of Soft Computing and Engineering, 2(6):504-510.

[7] Byungjun Son and Yillbyung Lee. 2005. Biometric authentication system using reduced joint feature vector of iris and face. In Audio-and Video-Based Biometric Person Authentication, pages 513-522. Springer.

[8] Calvin R Maurer and J Michael Fitzpatrick. 1993 A review of medical image registration. Interactive imageguided neurosurgery, 17, 1993.

[9] Ajita Rattani and Massimo Tistarelli. 2009 Robust multimodal and multiunit feature level fusion of face and iris biometrics. In Advances in Biometrics, pages 960-969. Springer.

[10] L Latha and S Thangasamy. 2010. A robust person authentication system based on score level fusion of left and right irises and retinal features. Procedia Computer Science, 2:111-120.

[11] Davide Maltoni, Dario Maio, Anil K Jain, and Salil Prabhakar. 2009 Handbook of fingerprint recognition. Springer.

[12] PP Chitte, JG Rana, RR Bhambare, VA More, RA Kadu, and MR Bendre. 2012. Iris recognition system using ica, pca, daugmans rubber sheet model together. International Journal of Computer Technology and Electronics Engineering, 2(1):16-23.

[13] Sunil Chawla and Aashish Oberoi. 2011 A robust algorithm for iris segmentation and normalization using hough transform. Global Journal of Business Management and Information Technology, 1(2):69-76.

[14] Zhenhua Guo, Lei Zhang, and David Zhang. 2010 Rotation invariant texture classification using lbp variance (lbpv) with global matching. Pattern recognition, 43(3):706-719.

[15] Anil K Jain, Arun Ross, and Salil Prabhakar. 2004 An introduction to biometric recognition. Circuits and Systems for Video Technology, IEEE Transactions on, 14(1):4-20.

[16] Philippe Parra. Fingerprint minutiae extraction and matching for identification procedure. University of California, San Diego La Jolla, CA, pages 92093-0443.

[17] BG Sherlock, DM Monro, and K Millard. 1994 Fingerprint enhancement by directional fourier filtering. In Vision, Image and Signal Processing, IEEE. Proceedings-, volume 141, pages 87-94. IET.

[18] Wuzhili. 2002 fingerprint recognition. 
[19] Heng Fui Liau and Dino Isa. 2011 Feature selection for support vector machine-based face-iris multimodal biometric system. Expert Systems with Applications, 38(9):11105-11111.

[20] Hugo Proenc, a and Luis A Alexandre. 2006. Iris recognition: An analysis of the aliasing problem in the iris normalization stage. In Computational Intelligence and Security, 2006 International Conference on, volume 2, pages 1771-1774. IEEE.

[21] Sunil Chawla and Aashish Oberoi. , 2011. Robust algorithm for iris segmentation and normalization using hough transform. Global Journal of Business Management and Information Technology, 1:69-76.

[22] Maeva Djoumessi. Iris segmentation \& recognition.

[23] John Daugman. How iris recognition works. 2004 Circuits and Systems for Video Technology, IEEE Transactions on, 14(1):21-30.

[24] Chong Siew Chin, Andrew Teoh Beng Jin, and David Ngo Chek Ling. , 2006 High security iris verification system based on random secret integration. Computer Vision and Image Understanding, 102(2):169-177.

[25] Shengcai Liao, Xiangxin Zhu, Zhen Lei, Lun Zhang, and Stan Z Li. 2007. Learning multi-scale block local binary patterns for face recognition. In Advances in Biometrics, pages 828-837. Springer.

[26] Alper Yilmaz and Muhittin G"okmen. 2001. Eigenhill vs. eigenface and eigenedge. Pattern Recognition, 34(1):181-184.
[27] Peter N. Belhumeur, Jo ao P Hespanha, and David Kriegman. 1997 Eigenfaces vs. fisherfaces: Recognition using class specific linear projection. Pattern Analysis and Machine Intelligence, IEEE Transactions on, 19(7):711-720.

[28] Chengjun Liu and Harry Wechsler., 2002 Gabor feature based classification using the enhanced fisher linear discriminant model for face recognition. Image processing, IEEE Transactions on, 11(4):467-476.

[29] Timo Ojala, Matti Pietik"ainen, and David Harwood. 1996 A comparative study of texture measures with classification based on featured distributions. Pattern recognition, 29(1):51-59.

[30] Timo Ojala, Matti Pietikainen, and Topi Maenpaa. 2002 Multiresolution gray-scale and rotation invariant texture classification with local binary patterns. Pattern Analysis and Machine Intelligence, IEEE Transactions on, 24(7):971-987.

[31] Xianbiao Qi, Yu Qiao, Chun-Guang Li, and Jun Guo. 2013 Multi-scale joint encoding of local binary patterns for texture and material classification.

[32] Timo Ahonen, Abdenour Hadid, and Matti Pietik"ainen. 2004 Face recognition with local binary patterns. In Computer vision-eccv 2004, pages 469-481. Springer.

[33] Prachi pooja godi smita thakre, kalyani. 2012. multimodal biometric feature based person classification. international journal of omputer application. 